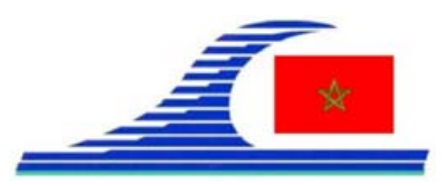

Conférence Méditerranéenne Côtière et Maritime

EDITION 2, TANGER, MAROC (2011)

Coastal and Maritime Mediterranean Conference

Disponible en ligne - http://www.paralia.fr - Available online

\title{
Le complexe lagunaire de Nador (Maroc) : fonctionnement, contrôle naturel et provoqué, scenarii d'évolution future
}

\author{
Naima HAMOUMI ${ }^{1}$ \\ 1. Laboratoire Océanologie et Géodynamique des Bassins sédimentaires, Faculté des \\ Sciences, Université Mohammed V - Agdal, Rabat, Maroc. \\ naimahamoumi@yahoo.fr
}

\section{Résumé :}

Le complexe lagunaire de Nador situé dans le littoral méditerranéen oriental (Maroc), présente un intérêt scientifique et socio-économique. L’étude de toutes les composantes de ce complexe lagunaire (masse d'eau, sédiments, microfaune) et de tous ses domaines physiographiques ainsi que son bassin versant et le proche plateau continental, a été menée à l'aide de disciplines et de techniques variées : sédimentologie, écologie, qualité de l'eau, étude morphostructurale, étude géomorphologie (basée sur l'étude de terrain et l'analyse de photo aériennes et d'images satellitaires), couplées à un suivi sur le terrain de l'état de l'environnement et une analyse des données historiques. Les résultats qui sont pour la plupart nouveaux et originaux, ont permis de comprendre le mode de genèse et de fonctionnement du complexe lagunaire et de mettre en évidence des évolutions d'ordre spatial et temporel à une échelle saisonnière et à l'échelle du millénaire. Ces évolutions sont liées à des facteurs naturels qui peuvent être interdépendants ou agir de concert et à des facteurs anthropiques. L'analyse de l'impact des facteurs naturels a également permis de prédire sept scenarii d'évolution future de cet écosystème.

En plus de leur intérêt scientifique, les résultats obtenus ainsi que les nombreuses cartes thématiques et les cartes SITO établies constituent une base de données importante et un outil de décision pour les aménagements de la région. Par ailleurs, la démarche proposée pour cette étude constitue un outil pratique pour l'exploitation et l'aménagement des écosystèmes littoraux en général et lagunaires en particulier.

\section{Mots-clés :}

Ecosystème côtier - Contrôle naturel et provoqué - Modèles d'évolution prédictif Lagune de Nador- Maroc - Littoral méditerranéen

\section{Introduction}

Le complexe lagunaire de Nador situé dans le littoral méditerranéen oriental (Fig. 1), est constitué par une lagune de $115 \mathrm{~km}^{2}$ de superficie dont la profondeur maximale atteint 7 $\mathrm{m}$ dans la partie centrale et une île barrière de $25 \mathrm{~km}$ de long dont la largeur varie de $300 \mathrm{~m}$ dans sa partie NW à $2 \mathrm{~km}$ dans son extrémité SE. Du fait de son intérêt scientifique et socio-économique, il a fait l'objet de nombreux travaux dans le cadre de 
La connaissance de la Mer :

un vecteur du développement durable en Méditerranée

la recherche académique et pour les besoins de l'aquaculture et de la pêche. Cependant, ces travaux ont été axés pour l'essentiel sur la lagune et ont porté sur les aspects: hydrologique, hydrochimique, microbiologique, sédimentologique, géochimique, écologique et micropaléontologique. Sachant que la genèse et l'évolution de ce complexe sont étroitement liées au contexte géologique régional et que ses différentes unités physiographiques sont interdépendantes, il était nécessaire d'entreprendre une étude qui intègre à la fois tous les domaines physiographiques et toutes ses composantes (masse d'eau, sédiments, microfaune) ainsi que son bassin versant et le proche plateau continental. Cette étude qui a été réalisée dans le cadre du projet COLASU, a pour objectifs de comprendre le mode de genèse et de fonctionnement ainsi que les différentes évolutions de ce complexe lagunaire afin de prédire son évolution future.

\section{Matériels et méthodes}

La démarche adoptée dans ce travail fait intervenir des disciplines et des techniques variées. En plus, d'une étude géomorphologique à l'échelle régionale faisant intervenir les données de terrain et l'analyse des photo aériennes au 1/20000 et des images satellites sur une période 30 ans (1975-2005), complétée par les données historiques et les résultats de l'analyse morphostructurale (LOUAYA \& HAMOUMI, 2010), il y a eu un suivi saisonnier durant la période 2003-2005 qui a porté sur : la sédimentologie, l'écologie (foraminifères benthiques et ostracodes), l'étude de la qualité des eaux lagunaires (surface et fond) et des eaux des émissaires de la bordure continentale (paramètres physico chimiques, éléments nutritifs, matière en suspension) et l'évaluation sur le terrain de l'état de l'environnement.

\section{Résultats}

La corrélation entre les milieux sédimentaires, les milieux écologiques et les zones hydrologiques et hydrochimiques reconstitués, a permis d'identifier les zones dynamiques de la lagune et de suivre leur répartition spatiale pour l'actuel (Fig. 1) et pour une période plus ancienne, estimée à 1000-1200 ans BP (Fig. 2) d'après les datations de MAHJOUBI (2001).

Il y a 1000 à 1200 ans BP, cet écosystème dont la limite continentale coïncidait avec la limite continentale actuelle du marais salant de Taouima (Zone VI, Fig. 2), correspondait déjà à une lagune de type restreinte avec huit zones dynamiques et une île barrière étroite et rectiligne de type microtidal dont la passe était située au niveau du double tombolo (Zone II, Fig. 2). Durant la période actuelle (2003-2005), la lagune ne compte plus que six zones dynamiques, cette évolution est liée essentiellement à une avancée de la bordure continentale suite à la transformation d'une partie de la lagune en marais (localité de Taouima) et à l'ouverture d'une passe en 1981 à 2 km au SE du double tombolo (Zone I, Fig. 1). Le contrôle saisonnier n'affecte que l'extrémité SE de la lagune où il s'exprime par un changement du milieu écologique. Le fonctionnement 
actuel du complexe lagunaire n'a d'ailleurs pas changé depuis quelques années comme le montre également les travaux précédents (BRETHES \&, TESSON, 1978 ; LEFEBVRE et al., 1996). Par ailleurs, la profondeur des eaux intralagunaires ne montre pas de variation sensible entre la période ancienne et la période actuelle. Les baisses eustatiques enregistrées au cours de l'actuel sont le résultat de l'effet conjugué de l'ensablement de la passe et de périodes de sécheresse. Ceci peut être expliqué par l'existence d'une subsidence continue (GUILLEMIN \& WERNLI, 1987 ; LOUAYA \& HAMOUMI, 2010) et de sa prédominance sur le taux de comblement (MAHJOUBI, 2001).

Cependant, l'équilibre actuel du complexe est menacé par les activités anthropiques qui se sont accrues et diversifiées ces dernières décennies. L’impact de la pollution continentale affecte la qualité de l'eau ainsi que la biodiversité et la densité de la microfaune, le comblement artificiel de l'extrémité NW accentue les processus de confinement. Enfin, l'aménagement de la passe effectué en 1995 a engendré un déséquilibre sédimentaire au niveau du littoral et de la lagune (HAMOUMI \& IRZI, 1999).

L'analyse de l'impact des facteurs naturels a montré que seuls la tectonique et le climat jouent un rôle prépondérant dans la genèse (transformation d'une baie) et l'évolution du complexe lagunaire de Nador. Elle a également permis de prédire sept scenarii d'évolutions futures faisant intervenir : la tectonique (exhaussement/ affaissement de la lagune et mouvements verticaux et/ou décrochants au niveau de l'île barrière), le volcanisme, les variations eustatiques et leur interaction.

\section{Conclusions}

Les résultats qui sont pour la plupart nouveaux et originaux, ont permis de mieux comprendre : la genèse du complexe lagunaire de Nador, son fonctionnement actuel et pour une période plus ancienne (1000 à 1200 ans BP), ainsi que les facteurs naturels qui ont contrôlent son évolution naturelle (à l'échelle saisonnière et à l'échelle du millénaire) et les facteurs anthropiques qui affectent son équilibre actuel. Ils ont également permis de prédire 7 scenarii d'évolutions futures de cet écosystème. En plus de son intérêt scientifique, la démarche proposée pour cette étude qui tient compte de l'évolution des processus morphostructuraux, géomorphologiques et sédimentologiques et de la qualité de l'eau ainsi que de leurs répercussions sur les systèmes vivants, constitue un outil pratique pour l'exploitation et l'aménagement des écosystèmes littoraux en général et lagunaires en particulier. Enfin, l'ensemble des résultats acquis ainsi que les nombreuses cartes thématiques et les cartes réalisées par les systèmes d'information territoriaux et opérationnels (SITO) constituent une base de données importante et un outil de décision pour les aménagements de la région. 
La connaissance de la Mer :

un vecteur du développement durable en Méditerranée

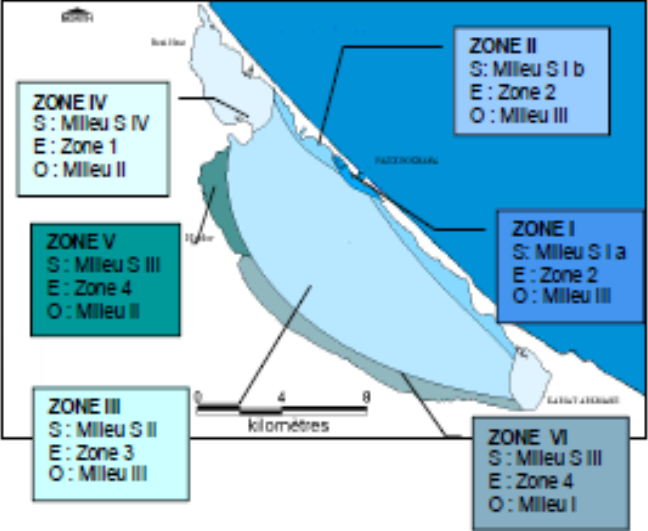

Figure 1. Les zones dynamiques de la lagune pour l'actuel.

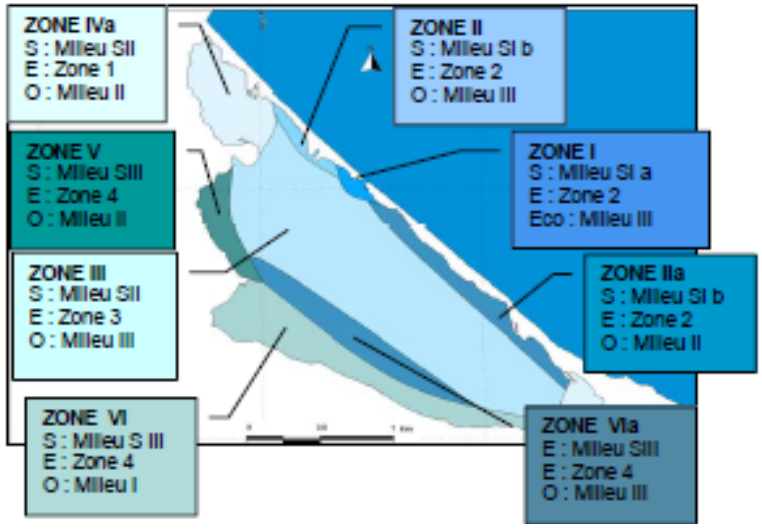

Figure 2. Les zones dynamiques de la lagune il y a 1000 à 1200 ans BP.

\section{Références bibliographiques}

BRETHES J.C., TESSON M. (1978). Observations hydrologiques sur la Sebkha Bou Areg (la lagune de Nador Maroc) Bilan d'automne 76 et d'hiver 77. Trav. Doc. Pêches Maroc, $\mathrm{n}^{\circ} 24$, Casablanca ; $17 \mathrm{p}$.

GUILLEMIN M., WERNLI R. (1987). Le sondage de Kariat Arkmane et le Néogène du bassin de Nador (Maroc oriental). Notes et Mém. Serv. Geol. Marco, Rabat, t. 43, $\mathrm{n}^{\circ}$ 321. pp 255-262.

HAMOUMI N., IRZI Z. (1999). Les aménagements côtiers : problèmes et enseignements - Cas du littoral méditerranéen oriental, Actes de la $3^{\text {ème }}$ Rencontre Hydrodynamique marine 99, 12 p.

LEFEBVRE A., GUELORGET O., PERTHUISOT J.P., DAFIR J. (1996). Evolution biogéologique de la lagune de Nador (Maroc) au cours de la période 1982-1993. Oceanologica Acta, 20, 2, pp 371-385.

LOUAYA A., HAMOUMI N. (2010). Etude morphostructurale de la région de Nador (Maroc nord-oriental). Africa Géosciences Review, Vol. 17, n² 2, pp 107-127.

MAHJOUBI R. (2001). Nature et origine du flux de matières particulaires et son enregistrement dans un milieu paralique microtidal : cas de la lagune de Nador (Maroc nord oriental). Thèse de Doctorat d’Etat ès Sciences, Univ Moulay Ismail, Meknès. $273 \mathrm{p}$. 